subjects in an independent validation set of serum samples.

The authors propose that the proteolytic degradation patterns in the serum peptidome might not only be used in cancer detection, but also to distinguish indolent from aggressive tumours. Such tests are urgently needed to identify men with prostate tumours who might safely avoid surgery or radiation therapy. The findings also indicate that proteomic analysis should not involve inhibition of proteolysis in ex vivo samples, which could limit biomarker discovery.

Kristine Novak, Nature Reviews Cancer

ORIGINAL RESEARCH PAPER Villanueva, J. et al. Differential exoprotease activities confer tumour-specific serum peptidome patterns.J. Clin. Invest. 116, 271-283 (2006)

FURTHER READING Coombes, K. R. et al. Serum proteomics profiling - a young technology begins to mature. Nature Biotechnol. 23, 291-292 (2005)

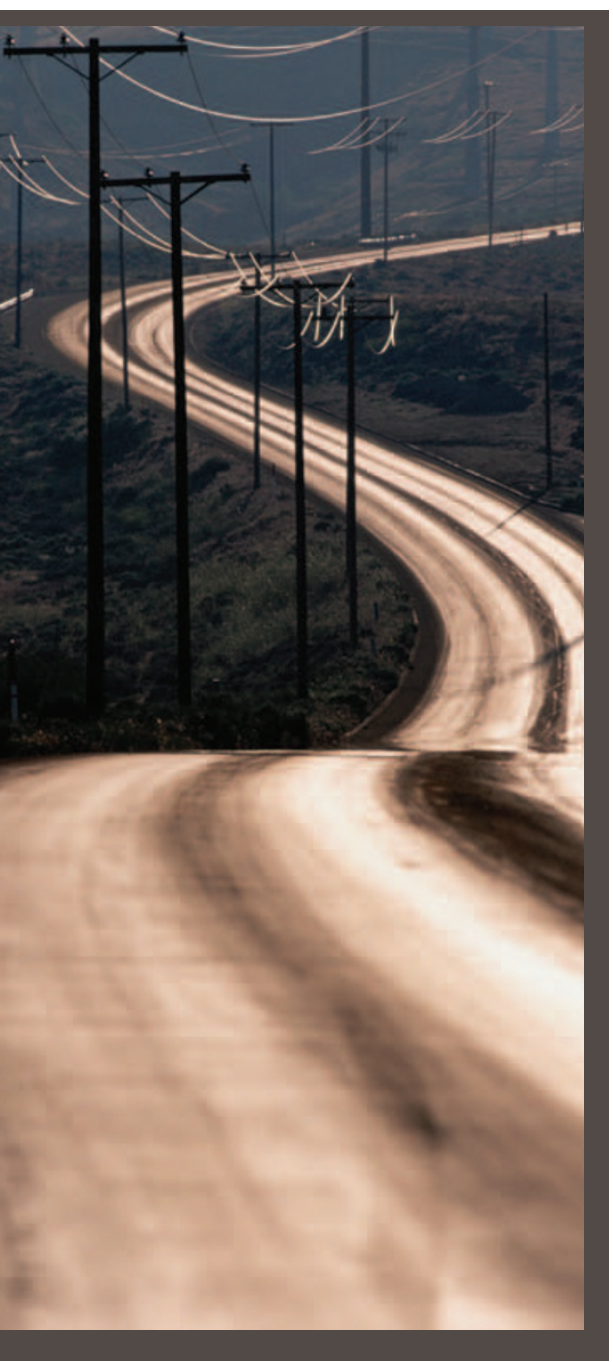

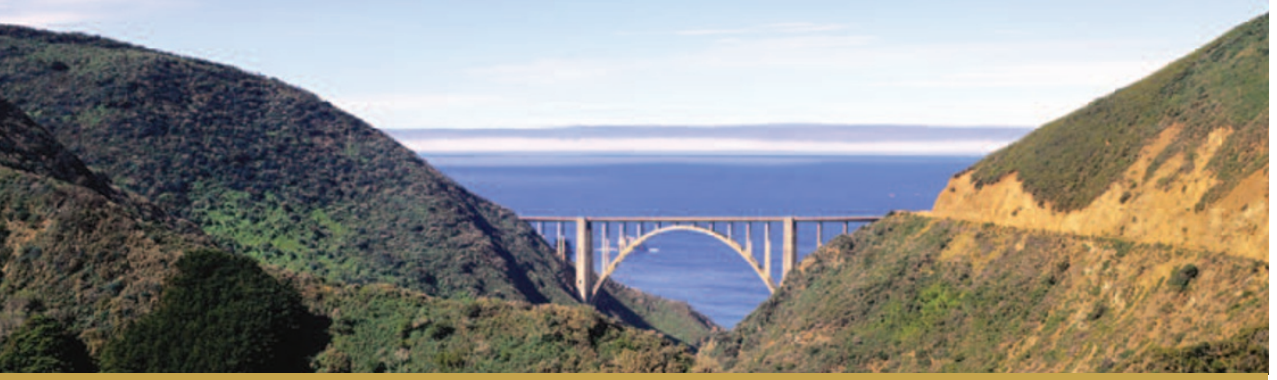

G-PROTEIN-COUPLED RECEPTORS

\title{
Bridging the GPCR gap
}

Evidence is accumulating for the existence and functional significance of GPCR heterodimers. Following the recent report that GPCRs form tissue-specific heterodimers in vivo, research just published in Proceedings of the National Academy of Sciences shows that bivalent ligands that target both $\mu$ - and $\delta$-opioid receptors have comparable analgesic benefit to morphine without the side effects chronic exposure causes, and could lead to the development of a new generation of improved analgesic drugs.

Research suggests that $\delta$-opioid receptors are involved in the development of tolerance and dependence to $\mu$-opioid receptor agonists, and studies with the $\delta$-antagonist naltrindole (NTI) have shown that the chronic side effects of morphine treatment can be blocked without reducing analgesia. Philip Portoghese and colleagues therefore proposed that a series of bivalent ligands that could modulate both receptors might make improved analgesics with fewer side effects and would also provide a useful tool for studying receptor synergy.

The authors designed and synthesized a series of bivalent ligands, the MDANs $(\mu-\delta$ agonistantagonists), consisting of a $\mu$-agonist pharmacophore (oxymorphone) and a $\delta$-antagonist pharmacophore (NTI) linked by variable-length diamine spacers (consisting of 16 , 19 or 21 methylenes). They administered the compounds to mice by intracerebroventricular (i.c.v) injection and found that the length of the spacer had profound effects on receptor function, providing evidence for bridging activity between the $\mu$ - and $\delta$-receptors. The antinociceptive activity of the bivalent ligands was enhanced by increasing the distance between the pharmacophores, but no such increase in potency was seen for monovalent $\mu$-opioid ligands or when $\mu$-agonist and $\delta$-antagonist were co-administered. This suggests that the increased $\mu$-agonist potency results from receptor association rather than the presence of both ligands, and that a certain length of spacer is required to achieve optimal bridging.

It is also possible that the $\delta$-receptor exerts negative allosteric cooperativity on the $\mu$-receptor, and so the authors studied this by pretreating mice with the $\delta$-antagonist, NTI, which they speculated would improve potency. As expected, NTI increased the potency of the bivalent ligands compared with control ligands possibly by displacing the bound MDAN $\delta$-antagonist and enabling the $\mu$-opioid receptor to be liberated from the negative allosteric effect of the $\delta$-opioid receptor.

Measuring the $\mathrm{ED}_{50}$ value of a ligand can be indicative of the development of tolerance to a drug. It was therefore interesting to see that after pretreatment with NTI, the $\mathrm{ED}_{50}$ value for MDAN-19 and MDAN-21 was the same as the monovalent $\mu$-opioid ligand, suggesting that $\delta$-antagonism reduces the development of tolerance to $\mu$-opioid agonists. To further investigate this, the authors studied the development of tolerance and withdrawal after chronic i.c.v administration. Although none of the bivalent ligands caused tolerance or dependence to the same extent as morphine, MDANs with shorter spacers produced tolerance without physical dependence and those with longer spacers produced neither tolerance nor dependence. Moreover, the MDAN-21 ligand was 50 -fold more potent than morphine by i.c.v or intravenous administration yet does not cause tolerance or dependence, suggesting that there is promise for the development of bivalent ligands as a new generation of analgesic drugs that do not cause physical dependence or tolerance with chronic use. These findings suggest that as we learn more about how cooperativity and other mechanisms of GPCR behaviour mediate distinct pharmacological effects it will become feasible to design targeted GPCR drugs with fewer and less severe side effects for a variety of indications.

Joanna Owens

ORIGINAL RESEARCH PAPER Daniels, D. J. et al. Opioid-induced tolerance and dependence in mice is modulated by the distance between pharmacophores in a bivalent ligand series. Proc. Natl Acad. Sci. USA 102, 19208-19213 (2005)

FURTHER READING Owens, J. It takes two... Nature Rev. Drug Discov. 4, 627 (2005)

WEB SITE

Philip Portoghese's laboratory: http://www.pharmacy.umn.edu/ faculty/portoghese_philip/home.html 